\title{
Einfluss von Rasse und Laktationsnummer auf Eutermerkmale bei Milchkiihen
}

\author{
Summary \\ Title of the paper: Influence of breed and lactation number on udder traits of dairy cows \\ For using automatic milking systems (AMS) a distance of $33 \mathrm{~cm}$ between teat and floor and of $1.5 \mathrm{~cm}$ between \\ two backteats is necessary. To estimate the percentage of cows of the in Germany common breeds of Holstein- \\ Friesian (HF), Simmental (FV), Brown Swiss (BV), Gelbvieh (GV), Red Holsteins (RH) and SMR (Synthetic \\ breed out of Friesian, Jersey and Holsteins), which don't fit to the AMS, was the aim of this trial. \\ The interval between milking and measuring influenced the distance between the two teats highly significant \\ $(\mathrm{p}<, 01)$, whereas only a trend of a decreasing teat-height could be shown. \\ The repeatability of the different traits had been .94 for teat-height, .88 for distance between the teats and .56 for \\ teat-length. \\ There are highly significant differences between the teat-height. of HF $(46.37 \mathrm{~cm})$, whereas the breeds GV \\ (38.88) and FV (39.45) show a pronounced decreased distance between teat and floor. The number of the \\ lactation influenced the teat-height in the expected way, too. \\ The teat-distance is influenced as well by the breed, as by the lactation. Under the assumption that AMS needs \\ udder-measurements of $\geq 33 \mathrm{~cm}$ teat-height and $\geq 1.5 \mathrm{~cm}$ distance between the hind teats, these requirements are \\ not fulfilled by $9 \%$ of Simmentals and by $7 \%$ of the $\mathrm{HF}$.
}

Key Words: teat traits, breed differences of cows, repeatability of measurement

\section{Zusammenfassung}

Für die Anwendung automatischer Melksysteme sind eine Zitzenhőhe von $33 \mathrm{~cm}$ und ein Zítzenabstand von 1,5 $\mathrm{cm}$ erforderlich. Inwieweit die deutschen Rassen Holstein-Friesian (HF), Fleckvieh (FV), Braunvieh (BV), Gelbvieh (GV), Rasse Deutsches Rotbunt (RH) und Schwarzbuntes Milchrind (SMR) diese Anforderungen erfullen, war Ziel der vorliegenden Untersuchung.

Der Messzeitpunkt hat einen hochsignifikanten Einfluss auf den Zitzenabstand, für die Zitzenhöhe konnte hingegen mit zunehmendem Zeitabstand nur ein Trend zu niedrigerem Bodenabstand aufgezeigt werden. Die Wiederholbarkeit der Messung war fur die Merkmale Zitzenhöhe 0,94, Zitzenabstand 0,88 und Zitzenlănge 0,56.

Für die Zitzenhohe wurden hoch signifikante Differenzen zwischen der Rasse $H F(46,37 \mathrm{~cm})$ sowie Gelbvieh $(38,88 \mathrm{~cm})$ und Fleckvieh $(39,45)$ festgestellt. Die Laktationsnummer wirkt sich erwartungsgemäß auf die Zitzenhöhe aus, alte Kuhe haben mit $36,91 \mathrm{~cm}$ eine um $14 \mathrm{~cm}$ niedrigere Zitzenhöhe als die der I.Laktation.

Der Zitzenabstand wird ebenfalls sowohl durch die Rasse als auch die Laktationsnummer beeinflusst. Die Mindestanforderungen automatischer Melksysteme hinsichtlich Zitzenhöhe und -abstand werden von $9 \%$ der FVKahe und $7 \%$ der HF nicht erfullt.

Schlusselworter; Zitzenmaße, Rinderrassenvergleich, Wiederholbarkeit der Messung

\section{Einleitung}

Schon bei der Einfuhrung des maschinellen Melkens gewannen die Eutermerkmale für einen rationellen Melkvorgang an Bedeutung. Bereits 1951 wurde für einen störungsfreien Ablauf des Melkens ein Bodenabstand von mindestens $45 \mathrm{~cm}$ gefordert (ANDREAE, 1963). Bei weitergehender Automatisierung des Melkvorganges gewinnt neben dem Abstand zum Boden auch der Abstand der Zitzen an Bedeutung, da die 
einzelnen Zitzen eindeutig voneinander differenziert werden müssen, um die Melkbecher mit hoher Erfolgsquote ansetzen zu können. Da die Hinterzitzen bei einigen Kühen teilweise ganz aneinander liegen, kann dies zur Merzung dieser Kühe bei der Einführung des automatischen Melkens führen. Aufgrund gegenseitiger Beeinflussung ist es nach SOMMER et al. (1961) sinnvoll, Leistungsmerkmale bei der Auswertung von Eutermerkmalen mit einzubeziehen. Die in Deutschland verbreiteten wichtigsten Rassen werden in die Untersuchung mit einbezogen, da bislang ein Vergleich einzelner Rassen in einer Untersuchung nicht vorliegt.

In der vorliegenden Arbeit sollen die Daten von Eutermessungen auf den Einfluss von Rasse, Alter, Laktationsstadium untersucht werden. Darüber hinaus soll durch wiederholte Messungen der Einfluß des Meßzeitpunktes abgeschätzt werden.

\section{Material und Methoden}

Untersuchungsmaterial

In der vorliegenden Untersuchung wurden Messungen an 952 Kühen der Rasse Deutsches Fleckvieh (FV), 524 Kühen der Rasse Deutsches Braunvieh (BV), 149 Kühen der Rasse Deutsches Gelbvieh (GV), 502 Holstein-Friesian-Kühen (HF), 53 Kühen der Rasse Deutsches Rotbunt (RH) und 1481 SMR-Kühen mit HF-Verdrängungskreuzung durchgeführt. Die Datenerhebungen des Feldmaterials erfolgten in einem Zeitraum von 2 Jahren auf insgesamt 59 Praxisbetrieben.

Des weiteren wurden 48 Fleckviehkühe eines Betriebes in einem Abstand von 2, 6 , 8,5 und 11 Stunden nach dem Melken gemessen, um den Einfluss des Zeitabstandes zwischen Melkzeitpunkt und Messung zu ermitteln.

Tabelle 1

Zitzenmaße und deren Definition (Teat traits and its definition)

\begin{tabular}{cc}
\hline Merkmal & Ansatzpunkte \\
\hline Zitzenhöhe & Abstand zwischen der Basis der tiefsten hintersten Zitze und der Standflache \\
Zitzenlänge & Abstand zwischen der Basis und der Spitze einer hinteren Zitze \\
Zitzenabstand & Abstand zwischen den Innenseiten der beiden hinteren Zitzenspitzen \\
\hline
\end{tabular}

Neben den in Tabelle 1 aufgeführten Merkmalen wurde auf den Betrieben die Kuhkennnummer, die Rasse, das Meßdatum und der Abstand zwischen Melken und Messung erfasst. Anschließend wurden Informationen der Leistungspriffung, wie die Laktatationsnummer, der Laktationstag und die Tagesmilchmenge, jeweils zum Meßtag, in den Datensatz eingearbeitet.

Bei 311 der 524 gemessenen Braunviehkühe liegt für das Merkmal Zitzenabstand kein Meßwert vor, da dieses Merkmal erst nach ersten Messungen in die Untersuchung mit einbezogen wurde.

\section{Methoden}

Die Datenauswertung wurde mit der PC - Version des Programmpaketes SAS (Statistic Analysis System, 1990) durchgeführt.

Für die Ermittlung des Einflusses des Zeitabstandes zwischen Melkzeitpunkt und Messung auf die Eutermerkmale wurde folgendes Modell verwendet:

wobei

$$
Y_{i j k}=\mu+M_{i}+K_{j}+e_{i j k}
$$


$\mu=$ Erwartungswert von $\mathrm{Y}_{\mathrm{ijk}}$

$\mathrm{M}_{\mathrm{i}}=$ Einfluß der i-ten Messung, $\mathrm{i}=1-4$

$\mathrm{K}_{\mathrm{i}}=$ Einfluß der $\mathrm{j}$-ten $\mathrm{Kuh}, \mathrm{j}=1-48$

$\mathrm{e}_{\mathrm{ijk}}=$ Summe aller Resteffekte

Die Varianzanalyse des Feldmaterials wurde nach folgendem Modell für die einzelnen Eutermerkmale durchgeführt:

wobei

$$
\mathrm{Y}_{\mathrm{ijk}}=\mu+\mathrm{R}_{\mathrm{i}}+\mathrm{L}_{\mathrm{j}}+\operatorname{cov}_{\mathrm{A}}+\operatorname{cov}_{\mathrm{L}}+\mathrm{e}_{\mathrm{ijk}}
$$

$\mu=$ Erwartungswert von $Y_{i j}$

$\mathrm{M}_{\mathrm{i}}=$ Einfluß der i-ten Rasse, $\quad \mathrm{i}=1-6$

$\mathrm{K}_{\mathrm{i}}=$ Einfluß der $\mathrm{j}$-ten Laktationsnummer, $\quad \mathrm{j}=1-7$

$\operatorname{cov}_{\mathrm{A}}=\mathrm{Abstand}$ zwischen Melken und Messung als Covariable

$\operatorname{cov}_{\mathrm{L}}=$ Laktationstage als Kovariable

$\mathrm{e}_{\mathrm{ijk}}=$ Summe aller Resteffekte

Ergebnisse und Diskussion

Wiederholte Messungen

In der Tabelle 2 sind die LSQ - Mittelwerte der wiederholten Messungen aufgeführt.

Tabelle 2

LSQ - Mittelwerte wiederholter Messungen (LSQ-Means of the traits measured in short intervals)

\begin{tabular}{ccccc}
\hline Merkmal & \multicolumn{4}{c}{ Abstand zwischen Melken und Messung } \\
& 2 Stunden & 6 Stunden & 8,5 Stunden & 11 Stunden \\
\hline Zitzenhöhe & 44,93 & 45,10 & 44,79 & 44,71 \\
Zitzenlänge & $5,29^{\text {ab }}$ & $5,20^{\mathrm{a}}$ & $5,11^{\mathrm{a}}$ & $5,48^{\mathrm{b}}$ \\
Zitzenabstand & $7,38^{\mathrm{a}}$ & $8,19^{\mathrm{b}}$ & $9,25^{\mathrm{c}}$ & $10,36^{\mathrm{d}}$ \\
\hline
\end{tabular}

mit unterschiedlichen Buchstaben gekennzeichnete LSQ - Mittelwerte sind signifikant voneinander verschieden $(p<0,05)$

Mit zunehmenden Abstand zwischen Melken und Messung nimmt der Zitzenabstand signifikant zu. 2 Stunden nach dem Melken beträgt der Zitzenabstand 7,38 cm und vergrößert sich bis kurz vor dem nächsten Melken auf $10,36 \mathrm{~cm}$. Dieses Ergebnis führte dazu, dass bei der geplanten Untersuchung das Intervall zwischen Melken und Messung aufgezeichnet wurde. Die Unterschiede im Merkmal Zitzenlänge sind nur teilweise statistisch gesichert und nicht eindeutig gerichtet. Das Merkmal Zitzenhöhe ändert sich hingegen zwischen zwei Gemelken nicht signifikant. Die Differenz von $45,10 \mathrm{~cm}$ bei der Messung nach 6 Stunden und dem Mittelwert von 44,71 cm bei der Messung nach 11 Stunden ist rein zufällig. Mit Hilfe der wiederholten Messungen wurde eine Wiederholbarkeit von 0,94 für die Zitzenhöhe, von 0,56 für die Zitzenlänge und von 0,88 für den Zitzenabstand ermittelt. Anhand des Standardfehlers kommen JOHANNSON (1957) und WITT et al. (1967) zu dem Schluss, dass die Genauigkeit der Messung ausreicht und eine Wiederholungsmessung nicht notwendig ist. Aufgrund vorliegender Ergebnisse der Wiederholbarkeitsmesswerte ist zu schlussfolgern, dass die Messgenauigkeit der Merkmale Zitzenhöhe und Zitzenabstand ausreichend ist, die Zitzenlänge jedoch mehrfach gemessen werden soll. 
Auswertung des Feldmaterials

Die Ergebnisse der Varianzanalyse zeigen, dass mit Ausnahme der Kovariablen Abstand zwischen Melken und Messen auf das Merkmal Zitzenhöhe sowie der Kovariablen Laktationstage auf die Zitzenlänge sich alle fixen Faktoren hochsignifikant auf die Merkmale auswirken (Tab, 3).

Tabelle 3

Varianzanalyse des Feldmaterials (Analysis of variance of field test)

\begin{tabular}{|c|c|c|c|c|c|c|}
\hline Varianzursache & FG & $\begin{array}{c}\text { MAQ } \\
\text { Zitzenhøhe }\end{array}$ & FG & $\begin{array}{c}\text { MAQ } \\
\text { Zitzenlange }\end{array}$ & FG & $\begin{array}{c}\text { MAQ } \\
\text { Zitzenabstand }\end{array}$ \\
\hline Laktationsnummer & 6. & $10783^{m}$ & 6 & $33,3 \mathrm{~m}$ & 6 & $288^{101}$ \\
\hline Rasse & 5 & $4841^{\cdots}$ & 5 & $56,3^{\cdots \cdots}$ & 4 & $554^{\cdots *}$ \\
\hline Abstand (Melken-Messen) & 1 & $115^{\text {n.s. }}$ & 1 & $17,3^{\cdots \cdot}$ & 1 & $165^{\cdots *}$ \\
\hline Melktage & 1 & $492^{* * *}$ & 1 & $3,1^{\text {ns }}$ & 1 & $3253^{\cdots *}$ \\
\hline Rest & 3556 & 36 & 3557 & 0,8 & 3183 & 11 \\
\hline
\end{tabular}

Signifikanzgrenzen: n.s. nicht signifikant; *0 $p<0,001$

Tabelle 4

LSQ-Means für Rassen (LSQ-Means of breeds

\begin{tabular}{|c|c|c|c|c|c|c|}
\hline Merkmal & Fleckvieh & Braunvieh & Gelbvieh & $\mathrm{HF}$ & Rotbunt & SMR \\
\hline Zitzenhøhe & $39,45^{n}$ & $43,86^{b}$ & $38,88^{*}$ & $46,37^{d}$ & $45,24^{\text {cd }}$ & $44,78^{c}$ \\
\hline Zitzenlänge & $5,54^{c}$ & $4,92^{\circ}$ & $5,84^{d}$ & $4,92^{2}$ & $5,21^{b}$ & $5,04^{b}$ \\
\hline Zitzenabstand & $10,45^{c}$ & $8,37^{\prime \prime}$ & $8,88^{\circ \circ}$ & $8,38^{\circ}$ & $9,68^{\text {be }}$ & $8,42^{n}$ \\
\hline
\end{tabular}

mit unterschiedlichen Buchstaben gekennzeichnete LSQ - Mittelwerte sind signifikant voneinander verschieden $(p<0.05)$

Tabelle 5

LSQ - Means fur die Laktationsnummem (LSQ-Means for number of lactations)

\begin{tabular}{|c|c|c|c|c|c|c|c|}
\hline \multirow{2}{*}{ Merkmal } & \multicolumn{7}{|c|}{ Anzahl Laktationen } \\
\hline & 1 & 2 & 3 & 4 & 5 & 6 & $>6$ \\
\hline Zitzenhơhe & $51,03^{\circ}$ & $47,07^{b}$ & $44,39^{\mathrm{c}}$ & $42,75^{d}$ & $39,98^{\mathrm{r}}$ & $39,53^{c}$ & $36,91^{\prime}$ \\
\hline Zitzenlänge & $4,79^{\star}$ & $5,06^{b}$ & $5,19^{\circ}$ & $5,27^{\text {cd }}$ & $5,41^{d e}$ & $5,46^{\circ}$ & $5,53^{\circ}$ \\
\hline Zitzenabstand & $7,73^{\circ}$ & $8,26^{b}$ & $8,62^{\text {be }}$ & $9,04^{\text {ed }}$ & $9,47^{d e}$ & $9,79^{\text {ef }}$ & $10,39^{\prime}$ \\
\hline
\end{tabular}

mit unterschiedlichen Buchstaben gekennzeichnete LSQ - Miltelwerte sind signifikant voneinander verschicden ( $p<0,05$ )

Der fixe Faktor Rasse hat aufgrund der Ergebnisse der Varianzanalyse den zweitgrößten Einfluss auf das Merkmal Zitzenhöhe. Zwischen den Rassen bestehen ausgeprägte Unterschiede (Tab, 3). Vor allem bei den Zweinutzungsrassen Gelbvieh und Fleckvieh sind die Euter statistisch gesichert im Mittel $6 \mathrm{~cm}$ tiefer als bei den Milchrassen angeordnet, was mit neueren Untersuchungen von GULYAS und IVANCSIS (2001) übereinstimmt, die für Kreuzungstiere Fleckvieh*HF mit einem HF-Blutanteil von ca. 80\% eine Zitzenhöhe von 46,25 cm nachgewiesen haben. Da aufgrund einer geringen Heritabilität des Merkmales zwischen 0,15 und 0,28 (ANDREAE, 1963; GENGLER et al.,1997; JOHANNSON, 1957; SMITH et al., 1985; WITT et al., 1967) ein hoher Zuchtfortschritt in diesem Sekundärmerkmal nicht zu erwarten ist, ist trotzdem eine konsequente, kontinuierliche Zuchtarbeit innerhalb dieser Rassen notwendig, um eine Verbesserung des Euters hinsichtlich des Bodenabstandes zu erreichen.

Die Unterschiede in der Zitzenhöhe sind zum überwiegenden Teil auf den Einfluß der Laktationsnummer zurückzuführen (Tab. 5), wobei die Zitzenhöhe von der ersten bis ab der siebten Laktation um knapp ein Drittel verringert wird. Andere Untersuchungen 
kommen zu ähnlichen Ergebnissen. JOHANNSON (1957) stellt bei 742 Kühen eine Abnahme des Bodenabstandes von der ersten bis zur 7. Laktation um $13 \mathrm{~cm}$ fest, wobei die Unterschiede höchstsignifikant waren, ANDREAE (1963) beschreibt ebenfalls eine statistisch gesicherte Abnahme des Bodenabstandes innerhalb von 8 Laktationen um ein Drittel. Bei der Untersuchung von 799 Kühen in der ersten und der dritten Laktation ermitteln WITT et al. (1967) eine statistisch signifikante Verringerung des Bodenabstandes um 6,7 cm.

Aus diesen Ergebnissen ist zu folgern, dass eine konsequente Selektion von Jungkühen erforderlich ist, um eine hohe Nutzungsdauer aufgrund einer guten Euteraufhängung zu ermöglichen.

Die Zitzenlänge nimmt in spăteren Laktationen in geringem, nur teilweise statistisch gesichertem Ausmaß zu. WITT et al. (1967) stellen eine gesicherte Zunahme der Zitzenlänge von der ersten bis zur dritten Laktation um $0,9 \mathrm{~cm}$ fest, wobei der Anteil der Varianzkomponente Laktationsnummer an der Gesamtstreuung im Merkmal Zitzenlänge ebenfalls nicht hoch ist.

Der Zitzenabstand nimmt innerhalb von 7 Laktationen um 2,66 cm zu, wobei die Unterschiede zwischen den einzelnen Laktationen nur teilweise statistisch gesichert werden konnten. Die Untersuchungen von ANDREAE (1963) und WITT et al. (1967) kommen zu ähnlichen Ergebnissen. Es läßt sich also feststellen, dass ein Einfluss der Laktationsnummer und somit des Alters auf die Merkmale Zitzenlänge und Zitzenabstand bestätigt werden kann.

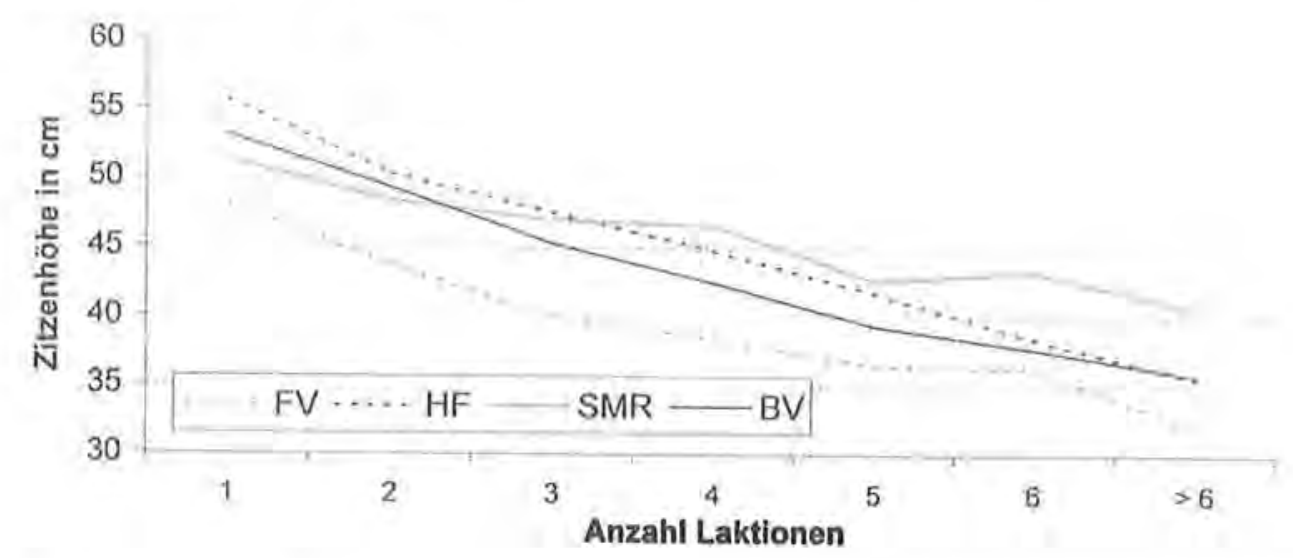

Abb. 1: Zitzenhohe in Abhangigkeit von Rasse und Laktationsnummmer (Teat height depending on breed and lactation number)

Abbildung 1 zeigt die Interaktion zwischen Rasse und Laktationsnummer im Merkmal Zitzenhöhe. Die in der 1. Laktation vorhandenen signifikanten Unterschiede zwischen den Rassen sind ab der 6. Laktation mit Ausnahme der SMR-Kühe, deren Zitzenhöhe mit den Laktationen nur vergleichsweise gering abfällt, nicht mehr vorhanden. Es zeigt sich, dass neben den Fleckviehkühen auch die älteren Kühe der Rassen Holstein Friesian und Braunvieh niedrige Zitzenhöhen aufweisen, was in der praktischen Melkarbeit zu Problemen führen kann. Die bei jungen Kühen der Rassen Holstein Friesian und Braunvieh vorhandene gute Euteraufhängung verschlechtert sich im Laufe der 
Laktationen wesentlich stärker als beim Fleckvieh und den sich hier sehr positiv hervorhebenden SMR-Tieren.

Auf das Merkmal Zitzenlänge hat der Faktor Rasse im Vergleich zu den anderen Faktoren den größten Einfluß. Da dieses Merkmal eine mittlere Heritabilität besitzt (SEYKORA et al., 1985; SMITH et al., 1985; SOMMER et al., 1961; WITT et al., 1967) und WÖRLE et al. (1988), lässt sich durch züchterische Maßnahmen relativ schnell eine Veränderung in die gewünschte Richtung erreichen. Es zeigen sich zwischen den Rassen teilweise statistisch gesicherte Unterschiede, wobei Braunvieh und Holstein Friesian-Tiere eher etwas kürzere Zitzen besitzen und die Fleckvieh- und Gelbviehtiere über dem Mittel liegen. Diese Unterschiede liegen aber in einem Bereich, in welchem sie auf die praktische Melkarbeit keinerlei Einfluss besitzen. Gegenüber früheren Untersuchungen hat sich die Zitzenlänge bei Braunvieh (SOMMER et al., 1961) von $6,5 \mathrm{~cm}$ auf $4,9 \mathrm{~cm}$ verringert. Gleiches gilt für Fleckvieh, wobei hier die Zitzenlänge von $7 \mathrm{~cm}$ (WÖRLE et al., 1988) auf $5,5 \mathrm{~cm}$ abnahm.

Das Merkmal Zitzenabstand besitzt nach WITT et al. (1967) eine mittlere Heritabilität. Die Rasse hat auf den Zitzenabstand einen mittleren Einfluss, der aber um einiges geringer ist als der der Laktationstage. Die Mittelwerte der untersuchten Rassen unterscheiden sich in diesem Merkmal nur wenig; nur der um 1,5 cm weitere Zitzenabstand der Rasse Fleckvieh weicht stärker von den anderen Rassen ab.

Die Beeinflussung des Merkmales Zitzenhöhe durch die Laktationstage ist nur sehr gering ausgeprägt. Innerhalb einer Laktation steigt die Zitzenhöhe um gut einen Zentimeter an, wobei dieser Wert aber mit einem mittleren Standardfehler von 0,00089 behaftet ist. WITT et al. (1967) stellen nur einen sehr geringen Einfluß des Laktationsstadiums auf die Zitzenhöhe fest. Ein geringer Anstieg der Zitzenhöhe zwischen der 6. und der 38. Laktationswoche konnte statistisch gesichert werden. Durch eine Heritabilitätsschätzung im Rahmen der obengenannten Untersuchung wird festgestellt, dass die Erblichkeit des Merkmals Zitzenhöhe im frischmelkenden Stadium am höchsten ist und dann im Laufe der Laktation abnimmt, da offenbar eine Reihe von Einflüssen nicht genetischer Art auf das Euter besteht, die zu einer Veränderung der Zitzenhöhe beitragen.

Das Laktationsstadium wirkt sich nicht auf die Zitzenlänge, jedoch hochsignifikant auf den Zitzenabstand aus. Er verringert sich statistisch gesichert innerhalb einer Laktation um knapp drei Zentimeter, wie aus Tabelle 6 zu entnehmen ist.

Tabelle 6

Regression der Eutermerkmale auf den Laktationstag (Regression of teat-traits on day of lactation)

\begin{tabular}{|c|c|c|}
\hline Merkmal & Regressionsgleichung & Standardfehler \\
\hline Zitzenhohe & $\mathrm{Y}=37,11+0,0033 \circ \mathrm{x}$ & $0,00089^{\cdots}$ \\
\hline Zitzenlänge & $\mathrm{Y}=5,61+0,00026 \cdot \mathrm{x}$ & $0,00013^{\mathrm{ns}}$ \\
\hline Zitzenabstand & $\mathrm{Y}=12,73-0,0089 \cdot \mathrm{x}$ & $0,00053^{\cdots}$ \\
\hline
\end{tabular}

WITT et al. (1967) können in ihrer Untersuchung ebenfalls eine gesicherte Abnahme des Zitzenabstandes von der 6. bis zur 38. Laktationswoche in einer Größenordnung von 2 bis $4 \mathrm{~cm}$ feststellen, wobei sie den Laktationstag als den wichtigsten Einflussfaktor auf das Merkmal Zitzenabstand bezeichnen. 
Anteil Kühe mit ungeeigneten Eutermaßen für Automatische Melksysteme (AMS).

Bei eigenen Voruntersuchungen an einem automatischen Melksystem wurde festgestellt, dass Mindestabstände von $33 \mathrm{~cm}$ Zitzenhöhe und 1,5 cm Zitzenabstand zum störungsfreien Melken benötigt werden. Daraus ergeben sich die in Abbildung 2 dargestellten rassenspezifischen Unterschiede.

Annähernd $9 \%$ der untersuchten Fleckviehkühe besitzen zu geringe Zitzenhöhen, was neben Problemen bei der Melkarbeit zu einem höheren Verletzungsrisiko der Zitzen und durch eine erhöhte Verschmutzung zu Hygieneproblemen führt. Braunvieh und Gelbvieh nehmen mit einer Häufigkeit von $5 \%$ eine Mittelstellung ein, wobei anzumerken ist, dass bei der Rasse Braunvieh vergleichsweise viele ältere Kühe gemessen wurden und im Gegensatz dazu bei der Rasse Gelbvieh nur für eine geringe Anzahl alter Kühe Messungen vorlagen. Bei Kühen der Rassen Holstein-Friesian und SMR treten zu geringe Zitzenhöhen nur mit einer Häufigkeit von $3 \%$ auf.

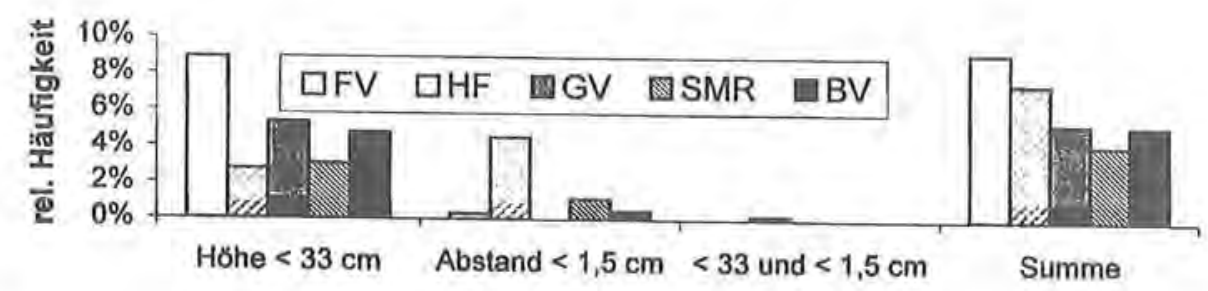
Abb. 2: Relative Haufigkeit ungunstiger Eutermerkmale (Frequency of teat measurements disqualifying for
AMS)

Beim Zitzenabstand zeigt sich, dass uber $4 \%$ der HF-Kuhe einen zu geringen Zitzenabstand von weniger als $1,5 \mathrm{~cm}$ aufweisen. Bei diesen Tieren ist zum einen kein optimaler Sitz des Melkzeuges gewährleistet, zum anderen können automatische Melksysteme die Zitzen nicht mehr voneinander unterscheiden. Nur bei den zu HF verwandten SMR - Tieren ist dieser zu geringe Abstand mit 1,2 \% noch von Bedeutung. Es ist aber anzumerken, dass durch Bewegungen der Tiere der Druck der Unterschenkel auf die prall gefullten Euterviertel verăndert werden kann, worauf der Zitzenabstand etwas variieren kann. Eine geringe Bewegung der Tiere kann also dazu fuhren, dass, obwohl die Zitzenspitzen vorher zu eng aneinander lagen, nur ein geringer, aber ausreichender Abstand zwischen den Zitzen entsteht und eine Erkennung hiermit möglich ist. Dies sollte bei der Beurteilung berücksichtigt werden. Extrem große Zitzenabstände uber $20 \mathrm{~cm}$, wie sie aber nur bei Ödemeutern nach der Geburt auftreten, behindern das Melken ebenfalls. Zitzenabstände von uber $20 \mathrm{~cm}$ wurden aber nur bei 14 von 3350 Tieren festgestellt, was einer sehr geringen Häufigkeit von $0,42 \%$ entspricht.

\section{Literatur}

ANDREAE, U.: Altersbedingte Veränderung von Euter- und Zitzenmaßen. Z. Tierzllcht. und Zuchtgsbiol, 78 (1963) 4 ,
$332-349$

BOETTCHER, P.J.; DEKKERS, J.C.M.; KOLSTAD, B.W.::

Development of an udder health index for sire selection based on somatic cell score, udder conformation and milking speed. J. Dairy Sci. 81 (1998) 4, 1157-1168 
GENGLER, N.; WIGGANS, G.R; WRIGHT, J.R.; NORMAN, H.D.; WOLFE, C.W.:

Estimation of $(\mathrm{Co})$ variance components for Jersey type traits using a repeatability model. J. Dairy Sci. $80(1997) 8,1801-1806$

GULYAS, L; IVANCSICS, J.:

Zusammenhănge zwischen der somatischen Zellzahl und einigen eutermorphologischen Eigenschaften. Arch. Tierz, Dummerstorf 44 (2001) 1, 15-22

JOHANNSON, I:

Untersuchungen uber die Variation in der Euter- und Strichform der Kuhe. Z. Tierzllcht. u. Zuchtgsbiol. 70 (1957), 233-270

JOHANNSON, I.; MALVEN, P.:

The Influence of Yield, Udder Pressure, Size of Teats and Teat Orifice on the Rate of Milking, Z. Tier-

SAS: zilcht. U. Zuchtgsbiol. 74 (1960), I-13

\section{SEYKORA, A. J.; MCDANIEL, B.T.:}

SAS7STAT User's Guide. Version 6. Fourth Edition (1990)

Heritabilities of Teat Traits and Their Relationships with Milk Yicld, Somatic Cell Count, and Percent Two-Minute Milk, J. Dairy Sci. 68 (1985), 2670-2683

SMITH, S.P.; ALLAIRE, F.R; TAYLOR, W.R; KAESER H.E.; CONLEY, J.:

Genetic Parameters Associated with Type Traits Scored on an Ordered Scale During Second and Fourth Lactation. J. Dairy Sci. 68 (1985), 2655-2663

SOMMER, O.A.; KRIPPL, J; FURTHMANN, G.N.:

Untersuchungen uber die Vererbung der Euter- und Zitzenformen beim Deutschen Fleckvieh und Deutschen Braunvieh. Bayr. Landw, Jahrbuch 38 (1961), 643 - 675

WITT, M.; LAUPRECHT, E.; ANDREAE, U.; FLOCK, D.:

Biometrische Untersuchungen an morphologischen Eutermerkmalen von frischmelkenden und altmelkenden Kuhen der ersten und dritten Laktation. Z. Tierzllcht. u. Zulchtgsbiol. 83 (1967), $11 \mathrm{II}-134$

WÖRLE, L; REINHARDT, F; WILLEKE, H.; PIRCHNER, F.:

Schätzung genetischer Parameter fur Hauptgemelk, maschinelles Nachgemelk und Eutermaße beim Fleckvieh. Zuchtungskunde, Stuttgart 60 (1988) 2, 85-97

Eingegangen: 18.11 .2000

Akzeptiert: 03.07.2001

Korrespondenzen an:

Prof. Dr. HENNING WILLEKE

Fachhochschule Weihenstephan

Abteilung Triesdorf

D-91746 Weidenbach-Triesdorf

E-Mail:henning.willeke@fh-weihenstephan.de 